\title{
On the geometry behind a recurrent relation
}

\section{CRistian Cobeli ${ }^{1}$ and Alexandru ZAHARescu ${ }^{1,2}$}

\section{ABSTRACT.}

We consider a certain linear recursive relation with integer parameters and study some of its algebraic and geometric properties, with the purpose of estimating the number of chains of valences in the Farey series.

Acknowledgement. The authors are grateful to the referee for his useful comments and suggestions.

\section{REFERENCES}

[1] Athreya, J. S. and Cheung, Y., A Poincaré Section for the Horocycle Flow on the Space of Lattices, Int. Math. Res. Notices, Vol. 2014 (2014), No. 10, 2643-2690

[2] Augustin, V., Boca, F. P., Cobeli, C. and Zaharescu, A., The h-spacing distribution between Farey points, Math. Proc. Cambridge Philos. Soc., 131 (2001), 23-38

[3] Badziahin, D. A. and Haynes, A., A note on Farey fractions with denominators in arithmetic progressions, Acta Arith., 147 (2011), No. 3, 205-215

[4] Boca, F. P., An AF algebra associated with the Farey tessellation, Canad. J. Math., 60 (2008), No. 5, 975-1000

[5] Boca, F. P., Cobeli, C., and Zaharescu, A., Distribution of lattice points visible from the origin, Comm. Math. Phys., 213 (2000), 433-470

[6] Boca, F. P., Cobeli, C. and Zaharescu, A., A conjecture of R. R. Hall on Farey points, J. Reine. Angew. Math., 535 (2001), 207-236

[7] Boca, F. P., Cobeli, C. and Zaharescu, A., On the distribution of the Farey sequence with odd denominators, Michigan Math. J., 51 (2003), 557-573

[8] Boca, F. P., Gologan, R. N. and Zaharescu, A., On the index of Farey sequences, Q. J. Math., 53 (2002), No. 4, 377-391

[9] Boca, F. P. and Zaharescu, A., The correlations of Farey fractions, J. London Math. Soc. (2), 72 (2005), No. 1, 25-39

[10] Boca, F. P. and Zaharescu, A., Farey fractions and two-dimensional tori, Noncommutative geometry and number theory, 57-77, Aspects Math., E37, Vieweg, Wiesbaden, 2006

[11] Bruckheimer, M. and Arcavi, A., Farey series and Pick's area theorem, The Math. Intelligencer, 17 (1995), No. 4, 64-67

[12] Cobeli, C., Ford, K. and Zaharescu, A., The jumping champions of the Farey series, Acta Arith., 110 (2003), No. 3, 259-274

[13] Cobeli, C., Iordache, A. and Zaharescu, A., The relative size of consecutive odd denominators in Farey series, Integers Electron. J. Comb. Number Theory, 3 (2003), A7, 14 pp.

[14] Cobeli, C., Vâjâitu, M. and Zaharescu, A., A density theorem on even Farey fractions, Rev. Roumaine Math. Pures Appl., 55 (2010), No. 6, 447-481

[15] Cobeli, C., Vâjâitu, M. and Zaharescu, A., On the intervals of a third between Farey fractions, Bull. Math. Soc. Sci. Math. Roumanie (N.S.), 53 (101) (2010), No. 3, 239-250

Received: 30.09.2013; In revised form: 09.09.2014; Accepted: 22.09.2014

2010 Mathematics Subject Classification. 11B57, 11B37.

Key words and phrases. Farey sequences, tessellations, geometry of recurrent relations.

Corresponding author: Cristian Cobeli; cristian.cobeli@imar.ro 
[16] Cobeli, C., Vâjâitu, M. and Zaharescu, A., The distribution of rationals in residue classes, Math. Reports, 14 (64) (2012), No. 1, 1-19

[17] Cobeli, C., and Zaharescu, A., The Haros-Farey sequence at two hundred years, Acta Univ. Apulensis Math. Inform., 5 (2003), 1-38

[18] Cobeli, C., Vâjâitu, M. and Zaharescu, A., On the Farey fractions with denominators in arithmetic progression, J. Integer Sequences, 9 (2006), Article 06.3.4, 26 pp. (electronic)

[19] Haynes, A., A note on Farey fractions with odd denominators, J. Number Theory, 98 (2003), No. 1, 89-104

[20] Haynes, A., The distribution of special subsets of the Farey sequence, J. Number Theory, 107 (2004), No. 1, 95-104

1 "Simion Stollow" Institute of Mathematics of the Romanian ACAdemy

P.O. BOX 1-764, BUCHAREST 70700, ROMANIA

E-mail address: cristian. cobeli@imar.ro

E-mail address: zaharesceillinois.edu

2 Department of Mathematics

UNIVERSITY OF ILLINOIS AT URBANA - CHAMPAIGN

1409 WEST GREEN STREET URBANA IL 61801, USA

E-mail address: zaharesceillinois.edu 